\title{
Modeling and Control of a Simulated Flight of a Mini Helicopter Using Matlab/Simulink
}

\author{
Mohamed Yacine Chachou, ZhiWen Liu, ZhiGuo Zhou, Abdelali Benchalal, Chemseddine Zerfaoui
}

School of Information and Communication, Beijing Institute of Technology, China

fkdfkrx@gmail.com, \{zwliu \& zhiguozhou\}@bit.edu.cn, alibench20@yahoo.fr, msouraj@sina.com

\begin{abstract}
In this paper, we present modeling and control of a simulated flight of a mini helicopter using matlab/simulink. First, we are interested in the analysis of dynamic modeling of helicopters. Indeed, based on the hypotheses established beforehand, we obtain some simplifications of the model for the cases of low-speed flight and thus to a stationary flight. Therefore the full model could be simplified by a three-step approach, to get the mathematical model of our mini helicopter. Second, we conceive control laws for the established dynamic model using several techniques of control. So we start by an open loop control via a joystick, to validate the response of the model to the inputs commands, given by a user. After, we design a PID controller to stabilize the system on a stationary flight. Then, we try to apply a nonlinear control for the helicopter such as the linearizing control. Finally, an optimal linear control is designed based on the Kalman observer. The use of this study is giving a basic simulation platform for mini helicopter, and compares different control laws results.
\end{abstract}

Index Terms - Helicopter modeling, Open Loop control, PID Controller, Linearizing control, Optimal Linear control.

\section{Introduction}

Aerial robotics knows for several years' considerable interest in both private builders and research laboratories. This interest is motivated by recent technological advances that make it possible to design efficient systems endowed with real autonomous navigation capabilities to no prohibitive costs. Unlike to terrestrial mobile robots for which it is often possible to be limited to a kinematic model, the control aerial robots require knowledge of a dynamic model. The effects of gravity and aerodynamic forces are the main causes. These systems, for which the number of control inputs is less than the number of degrees of freedom, expressed by under-actuated. The control mechanism usually provides one or two control inputs for the dynamics of translation and two or three control inputs for the rotational dynamics.

The main objective, of most of the articles dealing the problems of modeling a helicopter drone, is to identify a linear model with 6 degrees of freedom through a recorded during series of some flight tests or wind tunnel data. Reference [1], largely covers the theory of helicopter modeling. The author explains all the details necessary for a helicopter model. Reference [2], present the rotor dynamics. NASA developed in the 80s a helicopter test model [3]. This publication does not describe the modeling effort but presents a model that is used in the simulation environment. Reference [4], presents one of the first validation of a comprehensive mathematical model of a standard helicopter. The authors describe the development of a dynamic model for the EH-101. This model has more than 1,600 distributed parameters.
During the last decade, also the design methodologies of control laws for UAV helicopters have been developed with the aim to stabilize their movement either on a path or around a fixed position. In literature, few well-documented case of a non-linear helicopter model used for controller design $[5,8]$. In several papers, the design is based on linear models [6, 7].

In our study, we first analyze the dynamic modeling of helicopters. Then, we design some controllers to command the model. So, a proportional integral derivative controller (PID controller [9]), is developed as a classic control. After that, the linearizing control is discussed, it represent a non-linear helicopter model control. Finally, an optimal linear control is proposed.

\section{Modeling the Helicopter}

Here, the movement of the helicopter is described. The helicopter is considered as a rigid body, which means that Newton's law of second order and the Euler equations of motion of rotation can be applied. And also, because in the dynamic model of the helicopter, the terms of the force and torque vectors acting on the helicopter are used, therefore, it is necessary to determine them. For this, and Based on the work done by [7], a block diagram is provided for obtaining different quantities Fig. 1. The modeling is done based on the following hypotheses:

- The helicopter can be treated as a rigid body with the total number of rotor rotation;

- The effect of the air flow induced produced by the main rotor on the fuselage the helicopter is negligible;

- We are interested in a mode of free flight at low speed and hover;

- There is a plane of symmetry along the axis ZX in the helicopter. Thus, $\mathrm{I}_{\mathrm{xy}}=\mathrm{I}_{\mathrm{yz}}=0$;

- There is a plane of symmetry of mass along the $\mathrm{YZ}$ axis on the helicopter, this implies $\mathrm{I}_{\mathrm{Xz}}=0$.

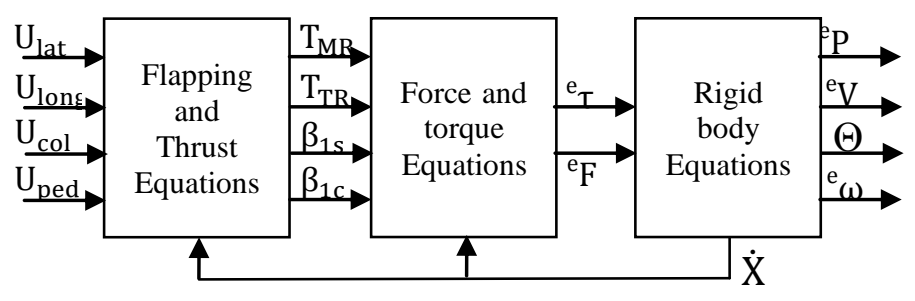

Fig. 1 Block Diagram for the Full Modeling of the Helicopter.

The principal system equations are given: 


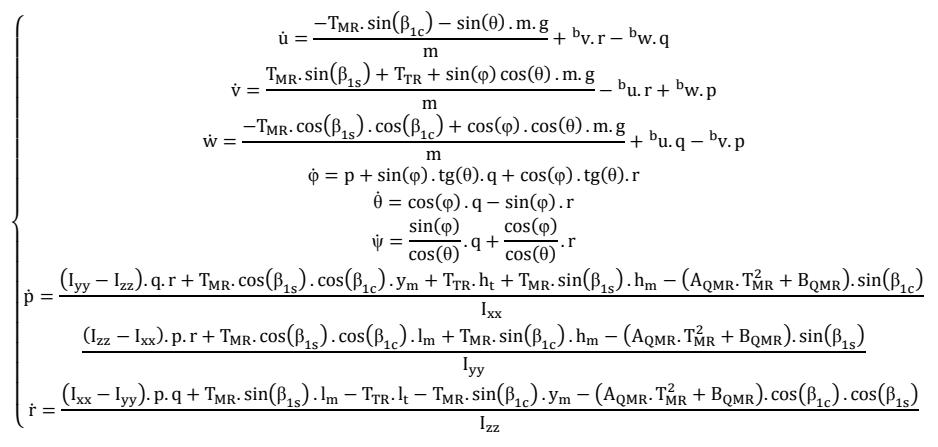

\section{Control and Simulation}

Once the full dynamic model is established, we are interested in the problem of control the helicopter drone. The helicopter is a complex nonlinear system with several variables, unstable in some flight ranges, and has a dynamic strongly coupled. In this section we will develop four kinds of control for the dynamic model developed previously. The first speaks about the open loop control via a joystick. That is to say, the orders will be injected by a user; this command allows us to validate the model. The second is control by PID (proportional, integral, derivative) in which we will choose the best gains that allow us to stabilize the helicopter by stabilizing the Euler angles. The third is a nonlinear controller which is the Linearizing control; it allows us decoupling our system. The fourth and final command is the optimal linear control. It means that our nonlinear system will be controlled by a linear control obtained after optimization of a performance criterion, and in which we will try to design a discrete controller that is based on a Kalman observer.

\section{A. Open Loop Control via a Joystick}

As mentioned earlier, this command will allow us to see more closely the behavior of the model. For this, a simulation environment MATLAB / SIMULINK has been developed, Fig. 2. This environment consists of three main blocks representatives the dynamic model of the mini helicopter, developed in the modeling section. It is also consists of a horizon display to see the orientation of the helicopter in space and a graphical interface for 3D visualization.

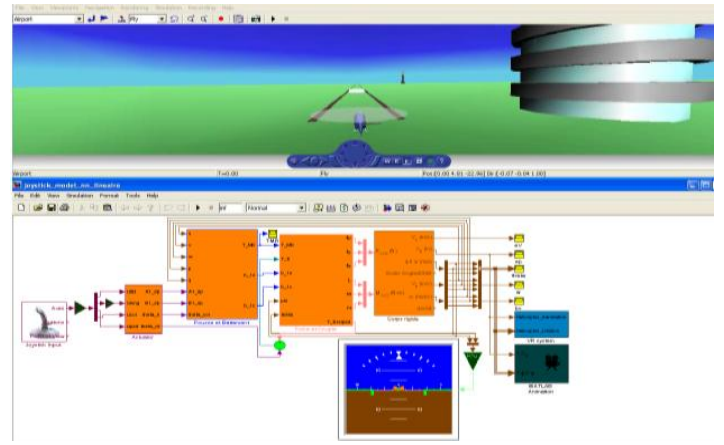

Fig. 2 Interface for control of the mini helicopter via Joystick.

For the test, we performed a horizontal front flight, and the obtained results are as follows:

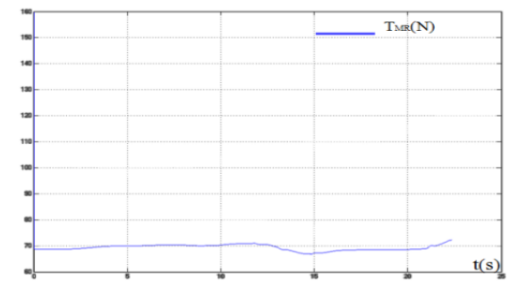

Fig. 3 The thrust of the main rotor.

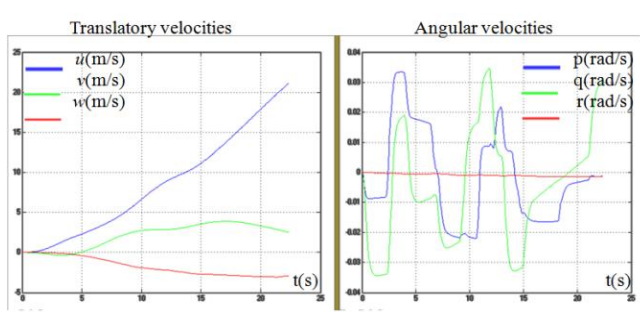

Fig. 4 Translatory and Angular Velocities.

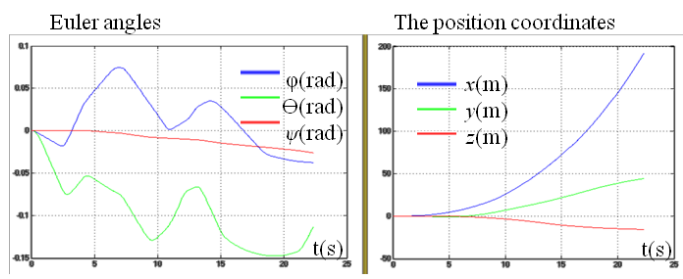

Fig. 5 Euler Angles and the Position Coordinates.

From the graph of the thrust, we note, at the beginning, a peak of amplitude equal to $160 \mathrm{~N}$, which represents the transitional regime. Then the value of this force decreases to $68.67 \mathrm{~N}$, it's the force that compensates the weight. Then, when we give the instruction to perform a forward horizontal flight, we see that the value begins to grow until it reaches the value of $70 \mathrm{~N}$, this allows the helicopter to gain in altitude. Then the value of the thrust fluctuates around $68.67 \mathrm{~N}$ because we tried to stabilize the helicopter. As we did a forward horizontal flight, the translatory velocities and the position, on the $\mathrm{x}$-axis, increase, this by making a pitch with negative value of $\theta$. It is confirmed in Fig. 4 (left) and Fig 5. We also note that the value of the translational velocity and position along the $y$-axis increases because of a roll movement and the same thing following the $\mathrm{z}$ axis, we can see and validate these observations from the final position in the following Figure:

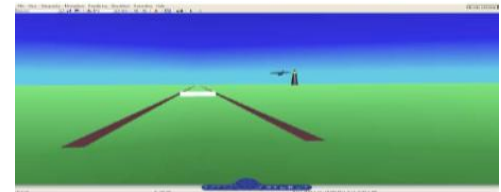

Fig. 6 Final position of the helicopter after a horizontal flight.

As remark, the values of the position and translational velocity along the z-axis appear decreasing; this is due to the orientation down of the z-axis.

From this test (a forward horizontal flight), we have been able to verify the validity of the dynamic model for an input 
command injected by a joystick. Hereafter, we will try to develop controls to stabilize the helicopter.

\section{B. PID Controller}

We propose in this section to develop a control, to stabilize the system, based on SISO PID controllers. That is to say that each input command will be controlled by a PID at a single input and a single output. Each PID input represents an Euler angle; therefore we will have at all three (3) controllers, Fig. 6 and Table I:

TABLE I Inputs and Outputs of each PID

\begin{tabular}{|c|c|c|}
\hline PID & Input & Output \\
\hline PID_phi & $\varphi$ & U lat \\
\hline PID_théta & $\theta$ & U $_{\text {long }}$ \\
\hline PID_psi & $\psi$ & U $_{\text {ped }}$ \\
\hline
\end{tabular}

The collective control input will be set to the value: $\mathrm{U}_{\mathrm{col}}=0.0861$ (rad) (this is the value that gives us the lift equal to the weight).

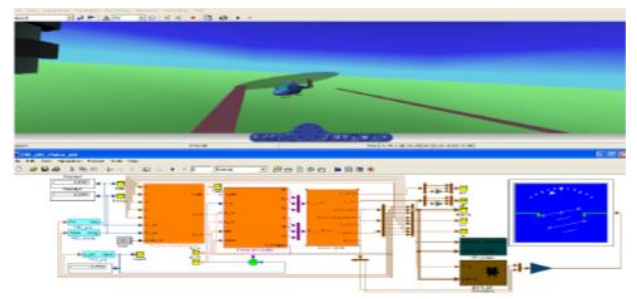

Fig. 7 Interface for PID Controller.

As the PID inputs are the Euler angles, the stability will be attitude stability. The procedure to adapting these three PID at the system was difficult due to the coupling between the state variables; they must be adjusted at the same time. As all controllers are PID type, therefore, the transfer function for each one is viewed as follows:

$$
\frac{S(p)}{U(p)}=K_{p}+\frac{K_{i}}{p}+K_{d} \cdot p
$$

Table II gives us a statement on the gain values used for attitude stabilization. These values are obtained by trial and error of the fact that the system is a multi input and output (MIMO), and the control strategy is to associate for each input, one output variable, it means a decoupled control.

TABLE II Gain Values for PID Controllers

\begin{tabular}{|c|c|c|c|}
\hline PID & $\mathrm{K}_{\mathrm{p}}$ & $\mathrm{K}_{\mathrm{i}}$ & $\mathrm{K}_{\mathrm{d}}$ \\
\hline PID_phi & 12.32 & 0.0006 & 0.72 \\
\hline PID_théta & -8.153 & 0.00937 & -0.654 \\
\hline PID_psi & 7.4 & 0.001 & 4.36 \\
\hline
\end{tabular}

Now we will try to stabilize the helicopter after we have imposed an initialization error on Euler angles, Equation 3, (see the position of the helicopter and the display horizon in Fig. 6).

$$
\left[\begin{array}{c}
\varphi \\
\theta \\
\psi
\end{array}\right]=\left[\begin{array}{c}
0.4(\mathrm{rad}) \\
0.3(\mathrm{rad}) \\
0.51(\mathrm{rad})
\end{array}\right]
$$

We are interested in the stability of the helicopter in the context of the Euler angles will be at zero. Hence the need to tend these destabilizing values, Equation 3, to the reference (zero). So the obtained results are:

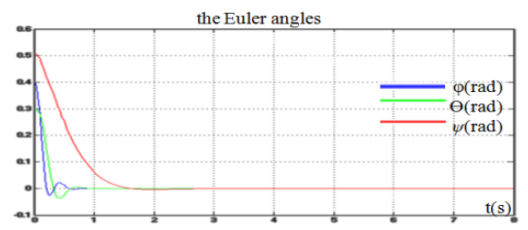

Fig. 8 The Euler Angles for PID Controller.

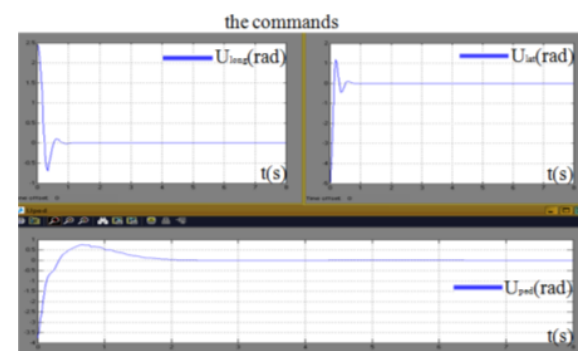

Fig. 9 The Inputs Commands for PID Controller.

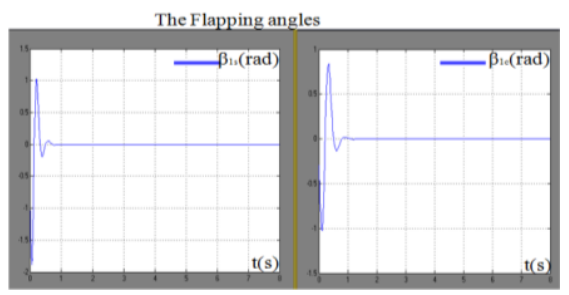

Fig. 10 The Flapping Angles PID Controller.

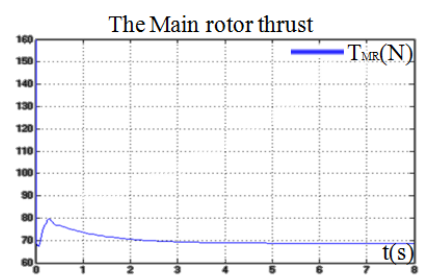

Fig. 11 The Main Rotor Thrust PID Controller.

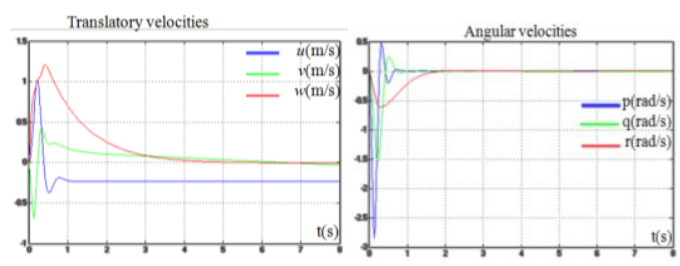

Fig. 12 Translatory and Angular Velocities PID Controller.

From Fig. 7, we see the convergence of Euler angles to zero which means that the three PID controllers, allow the system stability in the attitude. This can be confirmed by figures: angular velocities, the flapping angles, the main rotor thrust and the commands inputs. Because, when the control inputs converge to zero, they will automatically engage the convergence of the longitudinal and lateral flapping angles to 
zero. It means we will not have angular velocity along the lateral and longitudinal directions, and even the angular velocity, $r$, along the $z$-axis will be zero because the control on the pedals is also zero. All this makes the Euler angles converge on the position of stability in attitude which is zero. At the same time the thrust converges to the value $68.67 \mathrm{~N}$ to offset the weight and the helicopter remains stable on the altitude. Lacking doesn't take into consideration the control depending on the position and orientation of that system, the translation velocity is not really converges to the zero reference.

A second test is performed by taking the same initial values Equation 3 but with a negative sign. (See the position of the helicopter and the display horizon in Fig. 13). This test is done to validate the correct operation of PID controllers. The results are as follows:

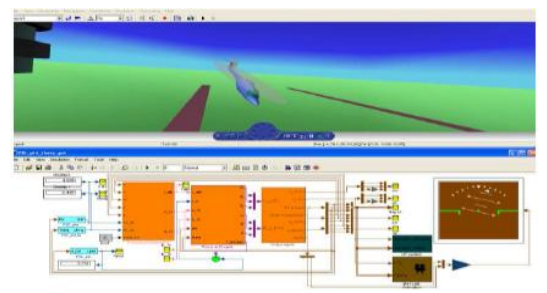

Fig. 13 Illustration of the initial position of the helicopter.

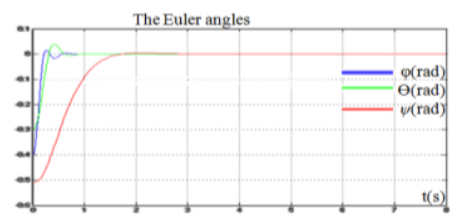

Fig. 14 Second Test Euler Angles.

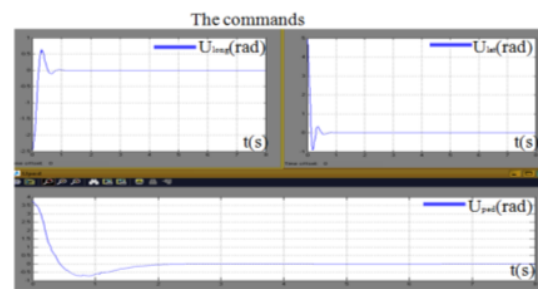

Fig. 15 Second Test Commands Inputs.

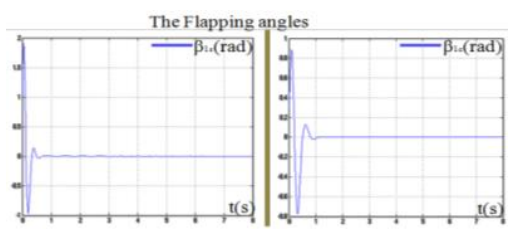

Fig. 16 Second Test Flapping Angles.

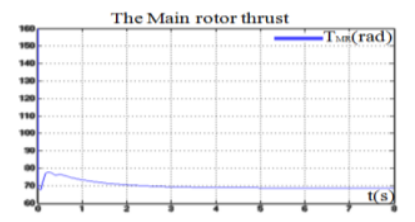

Fig. 17 Second Test Main Rotor Thrust.

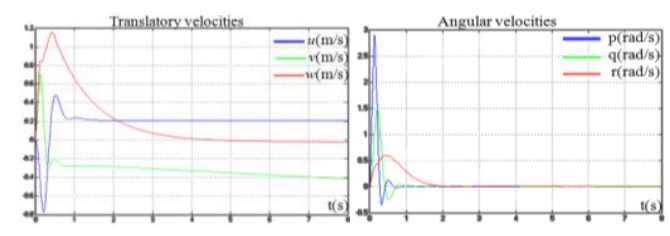

Fig. 18 Second Test Translatory and Angular Velocities.

From the obtained results we can confirm the functioning of three controllers for the attitude stabilization. But it is certain that there is no stability in the translational velocity to zero value. This problem can be solved by adding such position controller, for example the fuzzy controller.

To see the influence of gains to the convergence time of the system, a third test is scheduled, where we use the following values gains:

TABLE III Other Gain Values for PID Controllers

\begin{tabular}{|c|c|c|c|}
\hline PID & $\mathrm{K}_{\mathrm{p}}$ & $\mathrm{K}_{\mathrm{i}}$ & $\mathrm{K}_{\mathrm{d}}$ \\
\hline PID_phi & 13.32 & 0.6 & 1.325 \\
\hline PID_théta & -12.135 & 0.01 & -2.654 \\
\hline PID_psi & 7.4 & 0.001 & 4.36 \\
\hline
\end{tabular}

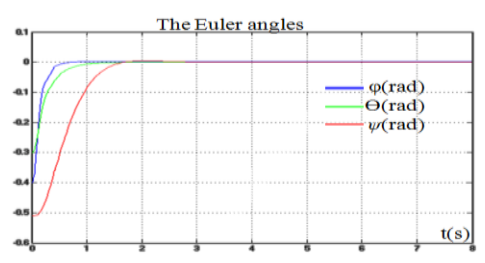

Fig. 19 The Euler angles for other gain values.

Since the control concerns the attitude, we will compare the convergence of Euler angles for the two cases, it means between the result of Fig. 14 and that of Fig. 19. For the first case we see that the angle $\varphi$ converges to the reference in time equal to 0.2043 (s), $\theta$ set a time of 0.4412 (s) and $\psi$ during $1.3322(\mathrm{~s})$. In the second case, $\varphi$ converges in $0.395(\mathrm{~s}), \theta$ in 0.762 (s) and $\psi$ in $1.3054(\mathrm{~s})$. So a priori the gain values given in Table 2 allow rapid convergence but when we see the shape of this convergence we see that there is appearance of oscillations, while for the shape of convergence achieved by the gain values given in Table 3 , there are no oscillations. So there is a tradeoff between the convergence time and the appearance of oscillations.

The results achieved by the attitude control using three standard PID controllers SISO are satisfactory since we have a stable system. Furthermore, we confirmed the existence of a compromise between having a minimum convergence time and the appearance of oscillations. But this command does not allow stability around a point, inter alia, a stationary flight.

\section{Linearizing control}

After establishing a Classic Control on the system, we will see in what follows the development of a nonlinear control for the helicopter, it concerns the linearizing control. The idea of linearizing control is to find a new basis for writing equations of the system (this basis is in the state space) 
such that the state equations become completely or partially linear. Thus we can synthesize the control law in the linear space and return to the original space. Based on the approach followed in [8], to apply the linearizing control on the model of the helicopter, simplifications must be taken into account namely the limitation to a hovering flight, consequently:

- The translatory and angular Velocities will be zero;

- The blade flapping can be neglected;

- The equations for generating the thrust become dependent only of the collective command;

- The vector product $\left({ }^{\mathrm{b}} \omega \times{ }^{\mathrm{b}} \mathrm{V}\right)$ can be neglected in the dynamics of fuselage (rigid body).

Relying on these simplifications, it can be seen that the dynamics of rotary wing (block thrust and flapping) is effectively decoupled from the rest of the dynamics. We can divide the model into two parts effectively decoupled, part that contains the generation of thrust and flapping and another part that contains the dynamics of a rigid body and the creation of the force and torque. The decoupled model will be as follows:

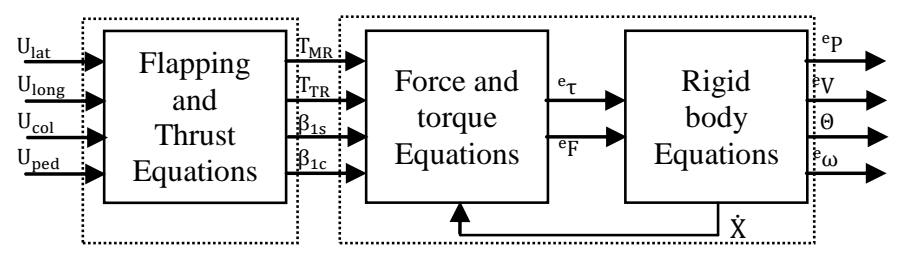

Fig. 20 Decoupled Helicopter Model.

So we will look at both blocks which contain the dynamic (state feedback), so the model is chosen to contain only the rigid fuselage dynamics and the dynamics of force and torque, therefore $\mathrm{T}_{\mathrm{MR}}, \mathrm{T}_{\mathrm{TR}}, \beta_{1 \mathrm{~s}}$ and $\beta_{1 \mathrm{c}}$ are regarded as inputs. The model becomes:

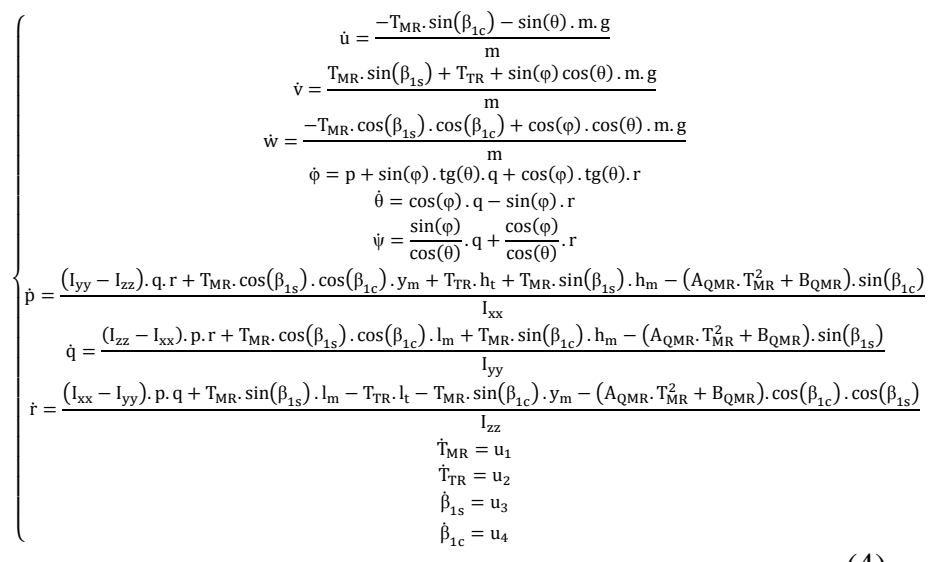

The purpose of the flight control is to design a helicopter autopilot of reduced model, so that the translational velocities and yaw angle follow a desired reference, $\mathrm{y}_{\mathrm{d}}=\left[\mathrm{u}_{\mathrm{d}}, \mathrm{v}_{\mathrm{d}}, \mathrm{w}_{\mathrm{d}}, \psi_{\mathrm{d}}\right]^{\mathrm{T}}$ it means that error $\left(\mathrm{e}_{\varepsilon}=\varepsilon-\mathrm{y}_{\mathrm{d}}\right)$ converges asymptotically to zero. Therefore, we take as output function $y=[u, v, w, \psi]^{T}$. This output function is interesting because it allows monitor path. With this option, the calculation of the relative degrees gives:

$$
\left\{r_{1}, r_{2}, r_{3}, r_{4}\right\}=\{2,2,2,3\}
$$

Unfortunately, the model of the helicopter has size (number of states) $\mathrm{n}=13$ and:

$$
r_{1}+r_{2}+r_{3}+r_{4}=9<13
$$

This implies the existence of an internal dynamic. To ensure the stability of the system, we check the stability of the four unobservable states by studying the zero dynamics [8].

To examine the stability of the system, a simulation environment was created, the output states of the system are initialized by the following values:

$$
\mathrm{y}=\left[\begin{array}{c}
\mathrm{u} \\
\mathrm{v} \\
\mathrm{w} \\
\psi
\end{array}\right]=\left[\begin{array}{c}
0.7(\mathrm{~m} / \mathrm{s}) \\
0.4(\mathrm{~m} / \mathrm{s}) \\
1.2(\mathrm{~m} / \mathrm{s}) \\
0.2(\mathrm{rad})
\end{array}\right]
$$

These values introduce an error on the reference, since we seeks the stability of the system, the state vector must be zero. The closed loop system should reduce the initial values, to the maximum. The results of the simulation are as follows:

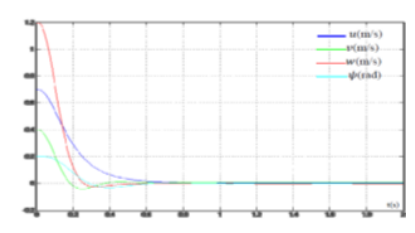

Fig. 21 The Outputs of the Controlled System (Observable State).

At first glance of the Fig. 21, simulated outputs appear to be stabilized, which presupposes that the control task was successful. However, the states of the zero dynamic, Fig. 22, show that the unobservable part of the system is far from stable.

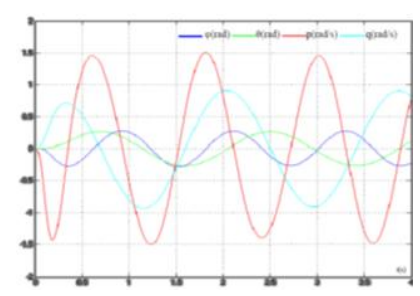

Fig. 22 The Zeros Dynamics (Unobservable State).

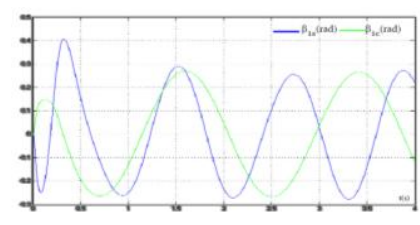

Fig. 23 The Flapping Angles.

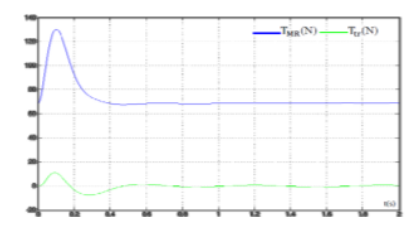

Fig. 24 The Thrust of Main and Secondary Rotors. 
We note that the variation of the flapping angle $\beta_{1 \mathrm{c}}$ is inversely proportional to the pitch angle $\theta$. Furthermore, $\beta_{1 \mathrm{c}}$ cannot be larger because of the structural limitations, such as the elasticity of the blade and the hinge saturation of swash plate. The thrust of the tail rotor converges to zero after 0.6 (s). It's the time taken by the yaw angle $\psi$ to also achieve stability. The thrust of the main rotor stabilizes after 0.4 (s) around the value $68.67(\mathrm{~N})$ corresponding to the value of the aerodynamic force to offset the weight $(\mathrm{mg})$.

To prove that the observed dynamics of the linearizing control system is stable even from the point of view, input limitations (saturation), we introduce these limitations under the form of saturation of hinges flapping with \pm 0.25 (rad) and we use the same initial conditions, we obtain the results:

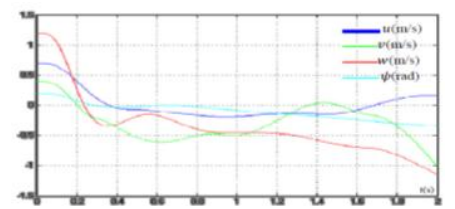

Fig. 25 The Outputs of the Controlled System in the Case of the Saturation of Hinges Flapping.

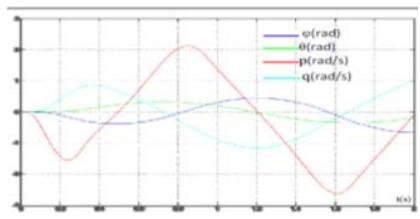

Fig. 26 The Zeros Dynamics in the Case of the Saturation of Hinges Flapping.

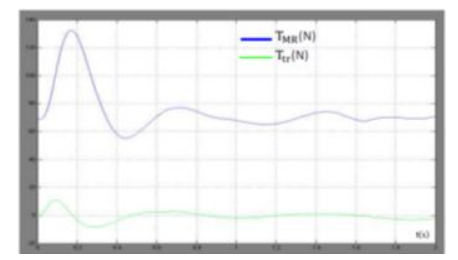

Fig. 27 The Thrust of Main and Tail rotors in the Case of the Saturation of Hinges Flapping.

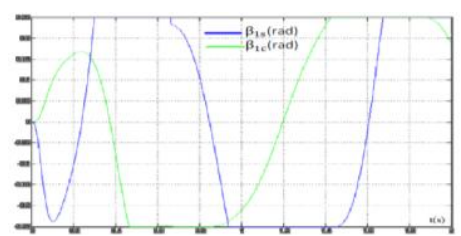

Fig. 28 The flapping Angles in the Case of the Saturation of Hinges Flapping.

Comparing the states of the closed loop system with saturation and states without saturation system, it can be seen that the outputs are decreasing for both systems until $\beta_{1 \mathrm{c}}$ reaching the saturation level, after; the closed-loop system is destabilized for the case of saturation on the hinges flapping. Also, the thrust of the main and secondary rotor are destabilized. Therefore we conclude that the exact linearizing control applied to the model of the helicopter is not possible. The study showed that the zeros dynamics is not asymptotically stable and consists at periodic orbits around 0 . Therefore, the standard helicopter is not a minimum-phase system due to the existence of the coupling between the rolling moment (resp. pitch) and lateral acceleration (resp. longitudinal).

\section{Optimal linear control}

Based on the study done by [7], the general objective of this control is to find an optimal control that minimizes a criterion which depends on the adopted orientation. The study of this approach contains two directions, the first orientation is a minimum energy while the second seeks to minimize the convergence time of the system.

First, we describe the design of a controller based on the optimal linear control which stabilizes the helicopter about an operating point and we estimate the operating range of state, based on the knowledge of the system open loop characteristic for the maximum state and input values can be used as starting point. For indeed, as illustrated in Fig. 29 open loop system initialized at the operating point is not stable.

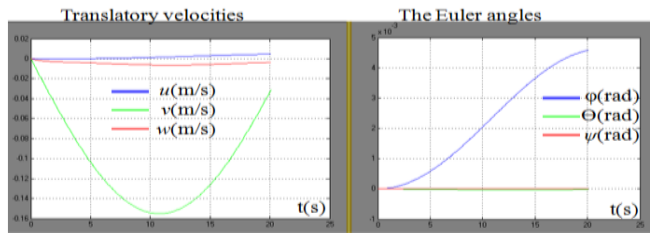

Fig. 29 Open Loop System Initialized at the Operating Point.

The goal with the first design stage is stabilization rather than optimizing performance, so the weighing will be performed on the following order of priority:

- Reduce the overshoot; attitude stabilization; and getting regular movement.

- Reduce the steady-state error on translatory velocities.

Considering these criteria, the results are as follows:

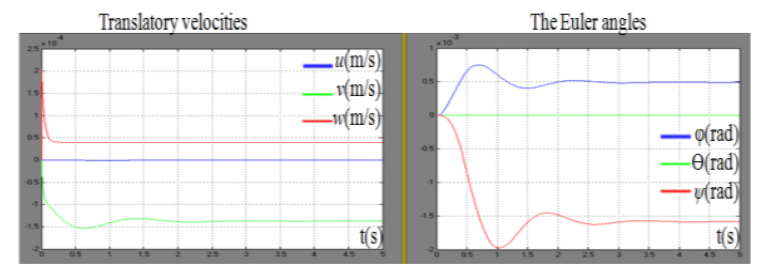

Fig. 30 Translatory velocities and Euler Angles for the First Design Stage of Optimal Control.

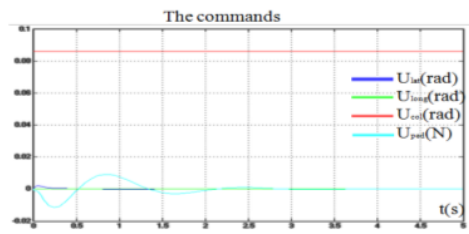

Fig. 31 Inputs Commands for the First Design Stage of Optimal Control. 


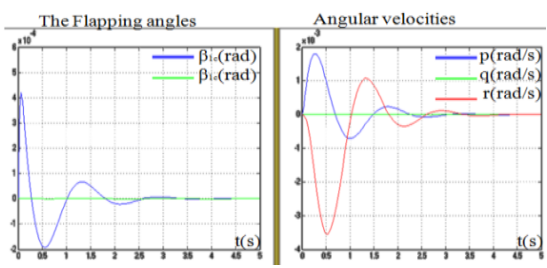

Fig. 32 Flapping Angles and Angular Velocities for the First Design Stage of Optimal Control.

From these simulations, we confirmed that the helicopter is stabilized with small errors on the translatory velocities. Comparing to [7], we have also find that the lateral velocity is dominant with approximately $1.4 \mathrm{~m} / \mathrm{s}$ steady-state error, due to the thrust of the tail rotor. We will treat this problem in that follows.

Second, to address the problem cited above, we may do the modeling of known disturbances. The errors on the translatory velocities are caused by errors of linearization which are not included in the controller design. However, it is possible to adjust these errors by introducing a model of disturbances in the control law [7]. By simulating the closed loop system with and without perturbation state, the obtained results are:

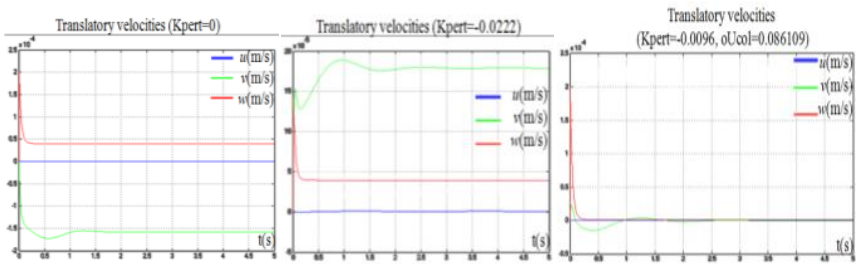

Fig. 33 Translatory Velocities after adjustment (Known Disturbances Modeling).

Where: $\mathrm{K}_{\text {pert }}$ a constant of perturbation.

Third, by introducing integral action in the controller, it is possible to eliminate the remaining errors. In addition, this improvement will also be adapted to the effects of all other external disturbances, constant, applied to nonlinear system [7]. The results are:

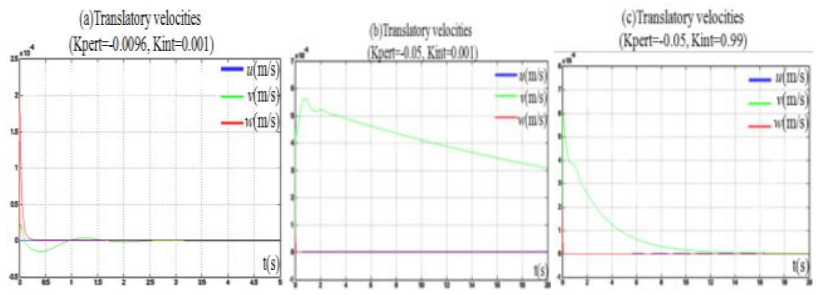

Fig. 34 The Translatory Velocities after adjustment (Integral Action).

Where: $\mathrm{K}_{\text {int }}$ a constant which toggles between an integral or proportional action.

From Fig. 34 (a) we see that when the perturbation is small we cannot see the effect of the integral action in the stabilization of the system. But when this perturbation becomes large, Fig. 34 (b, c), then the model with integral action is anyway stable. Merely, it's the value of $\mathrm{K}_{\text {int }}$ that makes the convergence will be fast or slow.
So far, during the controller design, complete information about the state are assumed, which is not the case in reality. Available sensors are three: accelerometers, three gyroscopes and a compass. This means that it is only possible to measure 7 of 11 statements. The flapping angles and two Euler angles $\theta$ and $\varphi$ are not directly measurable states and they must be estimated. So, in fourth, step, we design a Kalman observer that estimates not only the four absent states, but also do the evaluation of each of the 11 states [7]. After adjustment of Kalman Observer parameters, we obtained the following results:
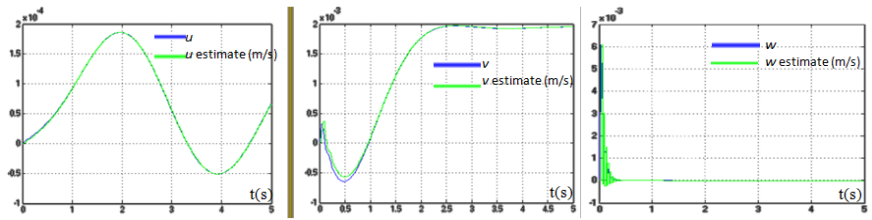

Fig. 35 Translatory Velocities Estimation (Kalman Observer).
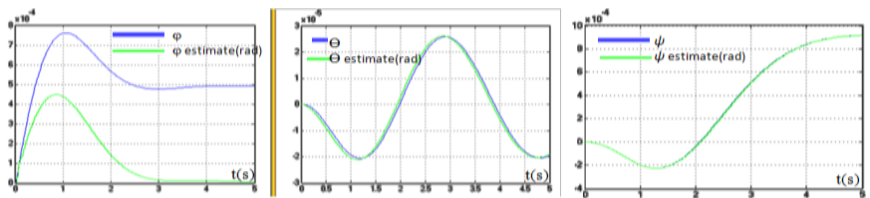

Fig. 36 Euler Angles Estimation (Kalman Observer).
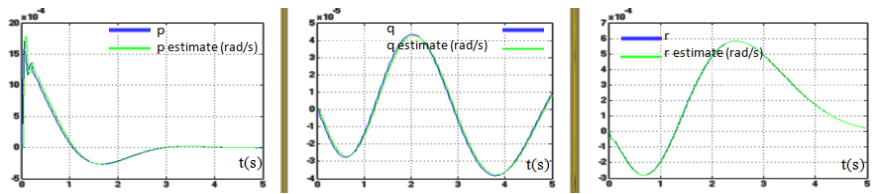

Fig. 37 Angular Velocities Estimation (Kalman Observer).
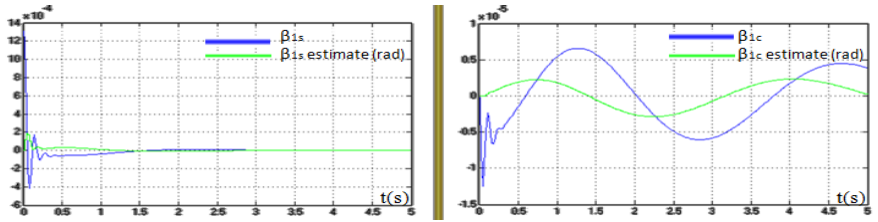

Fig. 38 Flapping Angles Estimation (Kalman Observer).

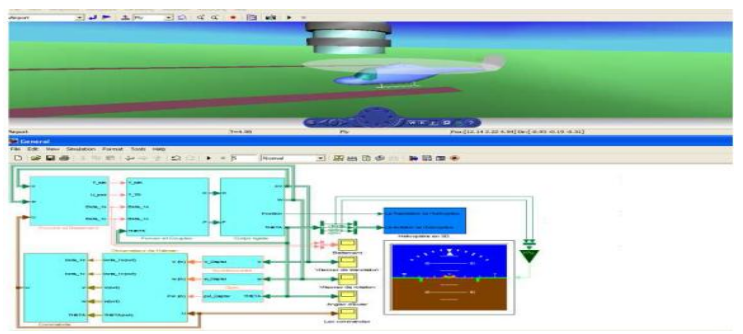

Fig. 39 Interface used for the whole optimal control.

The results show a good estimation of the states. The observer follows more exactly states of the system, except the flapping angles. That due to limitations of the linear model they are considered acceptable. The introduction of the observer causes some side effects that are oscillations in the 
velocities and the inputs commands. The reason for this can be found in the fact that the observer estimates the flapping angles from the linear model. We can remedy to this phenomenon by doing a tuning of the Controller [7].

The optimal linear control gives good results for the stability of the system around an operating point as long as tuning the Controller is done.

\section{Conclusions}

The work presented in this report has, initially focused on, obtaining a 6 DOF (Degree Of Freedom) nonlinear general model of a helicopter drone. Then, the model was used to free flight at low speed, and also for the design of control laws to stabilize it in stationary flight mode and offset the force of gravity and the dynamic effects of the two rotors.

The model was developed by using the Newton-Euler formalism. This model is considered complete, because, it takes into account of the dynamics of main rotor, namely the blade flapping and estimation of thrust depending on the induced velocity. However, it is not a general model since it does not take into consideration the case where the helicopter is subjected to a gust of wind, the effect of the soil and especially the deflection in the blade. The model thus established, has been validated in simulation by applying four kinds of control, open loop control via a joystick, a PID control, nonlinear control (the linearizing control) and an optimal linear control.

The open loop control was used to validate the dynamic analysis model. The next step has been devoted to the stabilization of the model around an operating point that is to say, obtaining a stationary flight. According to the obtained results, PID control provides good stability in attitude with a fast convergence. But with regard to stability around an operating point, it isn't assured, because the translational velocity does not converge to the zero reference. Regarding the exact linearizing control, it has been shown that the dynamics of zeros was not stable despite the convergence of observable states of the system. In addition, considering the limitations of the inputs (saturation), it was demonstrated that even observable states become destabilized. We conclude that the exact linearizing control is not applicable to helicopter model. Finally, the optimal linear control gave the best results for the stability in stationary flight.

\section{Acknowledgment}

We would like to acknowledge the significant contribution of AMRANE. F.

\section{References}

[1] W. Johnson, Helicopter Theory, Dover Publications inc, 1994.

[2] Leishman, J. Gordon, Principles of Helicopter Aerodynamics, Cambridge University Press, 2006.

[3] Heffley, K. Robert, and M. A. Mnich, Minimum-Complexity Helicopter Simulation Math Model, National aeronautics and space administration, Ames Research Center, 1988.

[4] A. Gee, T. Clarke, and P. Taylor, "A large scale helicopter model and its validation," European Control conference ECC'99, Karlsruhe, Germany, 1999.

[5] H.Ifassiouen, M. Guisser, and H. Medromi, "Robust nonlinear control of a miniature autonomous helicopter using sliding mode control structure,"
World Academy of Science, Engineering and Technology 2, vol. 3, no 3, p. 1, 2007.

[6] S. Carlos Mario Vélez, T. Andrés Agudelo, Colibri: a rapid prototyping methodology for the control of unmanned aerial vehicles, EAFIT University, June 15th, 2007.

[7] Hald, B. Ulrik, et al, "Autonomous helicopter-modelling and control," Report, Aalborg University 2005.

[8] Pettersen, Roger, E. Mustafic, and M. Fogh, "Nonlinear control approach to helicopter autonomy," Master Thesis, Aalborg University, 2005.

[9] M. S. Svenstrup, K.Winther, "Autonomous hover of an electric helicopter," Master Thesis, September 2006 - June 2007.

\section{Appendix}

TABLE IV Characteristics of the Helicopter

\begin{tabular}{|c|c|}
\hline $\mathrm{R}=0.77[\mathrm{~m}]$ & Rotor radius \\
\hline $\mathrm{R}_{\mathrm{l}}=0.415 / 2[\mathrm{~m}]$ & Radius (rotor center at beginning pallet) \\
\hline $\mathrm{y}_{\mathrm{m}}=0[\mathrm{~m}]$ & Distance center of mass to main rotor y-axis \\
\hline $\mathrm{l}_{\mathrm{m}}=0[\mathrm{~m}]$ & Distance center of mass to main rotor $\mathrm{x}$-axis \\
\hline $\mathrm{h}_{\mathrm{m}}=0.1[\mathrm{~m}]$ & Distance center of mass to main rotor z-axis \\
\hline $\mathrm{h}_{\mathrm{t}}=0[\mathrm{~m}]$ & Distance center of mass to tail rotor z-axis \\
\hline $\mathrm{l}_{\mathrm{t}}=0.6[\mathrm{~m}]$ & Distance center of mass to tail rotor $\mathrm{x}$-axis \\
\hline 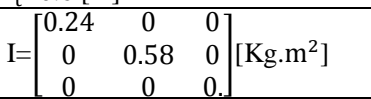 & The inertia matrix \\
\hline$\Omega=150[\mathrm{rad} / \mathrm{s}]$ & Rotor angular velocity \\
\hline$\rho=1.29\left[\mathrm{Kg} / \mathrm{m}^{3}\right]$ & Air density \\
\hline $\mathrm{a}=6[1 / \mathrm{rad}]$ & Polar slope (constant) \\
\hline $\mathrm{e}=0[\mathrm{~m}]$ & The hinge \\
\hline $\mathrm{c}=0.07[\mathrm{~m}]$ & Length of the cord \\
\hline$A=\pi \cdot R^{2}$ & Rotor disk surface \\
\hline $\mathrm{B}=2$ & Number of blades \\
\hline $\mathrm{m}=7[\mathrm{Kg}]$ & Helicopter weight \\
\hline $\mathrm{g}=9.81\left[\mathrm{~m} / \mathrm{s}^{2}\right]$ & Gravity \\
\hline$M_{b}=\frac{m \cdot R^{2}}{2} \cdot\left(1-\frac{e}{R}\right)^{2} \cdot g[N \cdot m]$ & Static moment \\
\hline$I_{b}=\frac{m \cdot R^{2}}{3} \cdot\left(1-\frac{e}{R}\right)^{3} \cdot g\left[K g \cdot m^{2}\right]$ & Inertia moment \\
\hline $\mathrm{e}_{\mathrm{MR}}=\frac{2.25}{12} \cdot 0[\mathrm{~m}]$ & Main-rotor hinge offset \\
\hline$\gamma=\frac{\rho \cdot \mathrm{a} \cdot \mathrm{c} \cdot \mathrm{R}^{4}}{\mathrm{I}_{\mathrm{b}}}$ & Blade lock number \\
\hline $\mathrm{R}_{\mathrm{CR}}=0.65 / 2[\mathrm{~m}]$ & Distance \\
\hline $\mathrm{R}_{\mathrm{CP}, \mathrm{p}}=\mathrm{R}_{\mathrm{l}}$ & Distance (rotor center, beginning pallet) \\
\hline$i_{s}=0$ & Initial inclination \\
\hline $\mathrm{K}_{\mathrm{MR}}=0.2$ & Linkage gain swashplate \\
\hline $\mathrm{K}_{\mathrm{CR}}=0.8$ & Linkage gain rotor control \\
\hline$\theta_{0}=0.0464[\mathrm{rad}]$ & $\begin{array}{l}\text { The first component Fourier series } \\
\text { development of the pitch angle }\end{array}$ \\
\hline$C_{r}=\frac{R \cdot c \cdot \Omega^{2} \cdot\left(R-\frac{e_{M R}}{2}\right)^{2} \cdot a \cdot \theta_{0}}{\pi \cdot R^{2} \cdot(\Omega \cdot R)^{2}}$ & Thrust coefficient \\
\hline $\mathrm{A}_{\mathrm{Q}, \mathrm{MR}}=0.000018$ & $\begin{array}{l}\text { Coefficient of ratio (main rotor thrust and } \\
\text { drag) }\end{array}$ \\
\hline $\mathrm{B}_{\mathrm{Q}, \mathrm{MR}}=0.01$ & The initial drag of main rotor \\
\hline $\mathrm{V}=[\mathrm{u}, \mathrm{v}, \mathrm{w}]^{\mathrm{t}}$ & Vector of translatory velocities \\
\hline$\Theta=[\varphi, \theta, \psi]^{\mathrm{t}}$ & Vector of Euler angles \\
\hline$\omega=[\mathrm{p}, \mathrm{q}, \mathrm{r}]$ & Vector of angular velocities \\
\hline${ }^{\mathrm{e}} \mathrm{P}[\mathrm{m}]$ & Position of the helicopter in earth landmark \\
\hline $\mathrm{T}_{\mathrm{MR}}$ & Thrust of the main rotor \\
\hline $\mathrm{T}_{\mathrm{TR}}$ & Thrust of the tail rotor \\
\hline$\beta_{1 \mathrm{~s}}$ & Lateral flapping angle \\
\hline$\beta_{1 c}$ & Longitudinal flapping angle \\
\hline $\mathrm{F}=\left[\mathrm{f}_{\mathrm{x}}, \mathrm{f}_{\mathrm{y}}, \mathrm{f}_{\mathrm{z}}\right][\mathrm{N}]$ & Force vector \\
\hline$\tau=[\mathrm{L}, \mathrm{M}, \mathrm{N}][\mathrm{N} . \mathrm{m}]$ & Torque Vector \\
\hline $\mathrm{U}_{\text {lat }}$ & Lateral input control \\
\hline $\mathrm{U}_{\text {long }}$ & Longitudinal input control \\
\hline $\mathrm{U}_{\mathrm{col}}$ & Collective input control \\
\hline$U_{\text {ped }}$ & Pedal input control \\
\hline
\end{tabular}

